\title{
Ultrasound Evaluation of Diaphragmatic Mobility and Contractility After Osteopathic Manipulative Techniques in Healthy Volunteers: A Prospective, Randomized, Double-Blinded Clinical Trial
}

\author{
Damiana Mancini, DO, ${ }^{a}$ Matteo Cesari, MD, PhD, ${ }^{\text {b }}$ Christian Lunghi, DO, ${ }^{\text {a,c }}$ \\ Augusto Maria Benigni, DO, PT, ${ }^{d}$ Raffaele Antonelli Incalzi, MD, ${ }^{e}$ and Simone Scarlata, MD ${ }^{f}$
}

\begin{abstract}
Objective: The purpose of this study was to investigate the effect of a session of osteopathic manipulative techniques on diaphragmatic motion and thickness in healthy participants.

Methods: This was a prospective, randomized, double-blinded, case vs sham vs control clinical trial performed in an outpatient osteopathic clinic in Rome, Italy. Sixty-seven healthy participants, mean age $40.4 \pm 14.5$ years, received an ultrasound evaluation of diaphragmatic motion and thickness, followed by a systematic osteopathic evaluation. After randomization, the experimental group $(n=22)$ received osteopathic manipulation, whereas the sham $(n=22)$ and the control $(n=22)$ groups had a light touch approach and simple observation, respectively. After a 1-session intervention, new osteopathic and ultrasound assessments were repeated in all participants.

Results: A statistically significant increase in diaphragmatic mobility was observed in the experimental group after the osteopathic manipulation $(\Delta=14.5 \mathrm{~mm}, P<.001$; analysis of variance $P<.001$ vs both sham: $\Delta=-0.22 \mathrm{~mm}$, and control: $\Delta=$ $-2.09 \mathrm{~mm}$ groups). A strong linear relationship was observed between the diaphragmatic motion gradient, measured with ultrasonography, and the score assigned by the operator evaluating the change of diaphragm mobility after intervention. Conclusion: Osteopathic techniques used in this study improved the diaphragmatic motion (but not the muscle thickness) in healthy participants. Further studies are needed to confirm our findings and eventually identify the clinical conditions that may benefit from osteopathic manipulative treatment of the diaphragm. (J Manipulative Physiol Ther 2019;42:47-54)

Key Indexing Terms: Manipulation, Osteopathic; Diaphragm; Respiration; Ultrasonography
\end{abstract}

${ }^{\mathrm{a}}$ Centro Ricerche Olistiche per la Medicina Osteopatica e Naturale (CROMON), Rome, Italy.

${ }^{\mathrm{b}}$ Gérontopôle, Centre Hospitalier Universitaire de Toulouse, Université de Toulouse III Paul Sabatier, Toulouse, France.

${ }^{c}$ Clinical-based Human Research Department, Research Division, C.O.M.E. Collaboration, Pescara, Italy.

${ }^{\mathrm{d}}$ European Osteopathic Project (EOP), Scuola Alain Bernard, Rome, Italy.

${ }^{\mathrm{e}}$ Division of Geriatrics - Campus Bio Medico University and Teaching Hospital, Rome, Italy.

${ }^{\mathrm{f}}$ Unit of Respiratory Pathophysiology and Thoracic Endoscopy, Division of Geriatrics, Campus Bio Medico University and Teaching Hospital, Rome, Italy.

Corresponding author: Simone Scarlata, MD, Unit of Respiratory Pathophysiology and Thoracic Endoscopy, Campus Bio Medico University and Teaching Hospital, Via Alvaro del Portillo 200, 00128 Rome, Italy. Tel.: +39 06225411167.

(e-mail:s.scarlata@unicampus.it).

Paper submitted August 30, 2017; in revised form June 6, 2018; accepted August 16, 2018.

0161-4754

Copyright (C) 2019 by National University of Health Sciences. https://doi.org/10.1016/j.jmpt.2018.08.001

\section{INTRODUCTION}

The diaphragm is considered the main muscle of respiration and plays a vital role in the breathing process. The complex anatomic and biomechanic nature of the thoracic diaphragm, as well as its multiple functions in the human body function, provide a rationale for osteopathic manual intervention. The diaphragm muscle assists in respiration, but also has many roles connected to the health of the body. It is important for posture, for proper pelvic and oral (ie, both for phonation and swallowing) functions. It also stabilizes the cervical spine and thoracic outlet and is important for the vascular and lymphatic systems. ${ }^{1}$

Diaphragm movement may influence the movement of the heart because the pericardial sac is connected to the diaphragm by phrenic-pericardial ligaments ${ }^{2}$ and affects vena cava collapsility $^{3}$ and venous return to the heart. ${ }^{4}$ Furthermore, diaphragmatic motion is known to contribute to increasing the heart rate during inhalation. ${ }^{5}$ Degenhardt and Kuchera ${ }^{6}$ describe how pressure gradients created by the movement of the diaphragm can influence lymphatic flow dynamics and affect gastrointestinal and cardiovascular systems. Lymph flow 
is therefore influenced by myofascial compression, and this is consistent with the basic osteopathic concepts relating to homeostasis and the inter-relationship of body systems. ${ }^{7}$

Traditional osteopathy, according to the World Health Organization and the World Osteopathic Health Organization, is defined as

a natural medicine which aims to restore physiological function and homeostasis in the body by treating the somatic (body framework) dysfunction, that is, impaired or altered function of related components of the somatic system. To achieve this goal the Osteopathic Manual Practitioner relies on the quality and finesse of his/her palpation and works with the position, mobility and quality of the tissues. ${ }^{8,9}$

Although a number of osteopathic techniques focusing on the diaphragm have been proposed and commonly practiced within the osteopathic profession, there is a general lack of evidence to identify if osteopathic manipulative techniques on the diaphragm are effective and able to modify the muscle's contractility and motion.

In a recent study, da Silva and colleagues compared manometry pressures of the lower esophageal sphincter before and immediately after osteopathic intervention in the diaphragm of a participant with gastroesophageal reflux, finding a beneficial increase soon after the intervention. ${ }^{10}$ The estimation of the osteopathic manipulative treatment (OMT) effectiveness on diaphragmatic activity, however, did not provide any information about changes in motility and contractility in that study.

In another double-blinded, randomized, controlled trial, 406 hospitalized patients with pneumonia were randomized to receive conventional care only, light touch treatment, or OMT (including the doming technique of the diaphragm), in adjunction to conventional treatment. The study showed a significantly shorter length of stay in the OMT and light touch groups compared to the control group. However, this study reported no objective evaluation was available documenting the benefits of the OMT on diaphragmatic activity. ${ }^{11}$

The effect of OMT has also been investigated in chronic obstructive pulmonary disease (COPD) patients with contrasting results, which may be due to the heterogeneity of the manual therapy used, the number of the intervention sessions (single vs multiple), and the age of the patient $(<50$ years compared to $>50$ years). A study of 35 participants using a single application of an OMT protocol based on 7 standardized manipulative techniques that included indirect myofascial re-doming of the diaphragm was associated with an overall worsening of air trapping during the 30 minutes immediately after the procedure. ${ }^{12}$ In contrast, other studies reported improvements in lung function in severe COPD patients after OMT. ${ }^{13-15}$

Hand-held ultrasound instruments allow for the morphology and function of the diaphragm to be assessed in different settings and under different conditions. ${ }^{16}$ Ultra- sonography is currently the only noninvasive, nonionizing imaging technique widely available to directly assess diaphragmatic function. Two different sonographic approaches allow the assessment of muscle thickening in the zone of apposition and excursion of the dome of the diaphragm. ${ }^{17}$

The purpose of this study was to investigate the effects of a single session of osteopathic manipulation of the diaphragm on muscle motility and thickness, assessed by diagnostic ultrasound, in a sample of healthy participants.

\section{Materials and Methods}

\section{Design of the Study}

A prospective, randomized, double-blinded case vs sham vs control trial was conducted (allocation ratio 1:1:1). The eligibility criteria included asymptomatic healthy adults, absence of chronic pain or acute symptomatology during the last 72 hours before the session, and no diagnosed pathology.

Participants presenting the following conditions were excluded from the trial: pregnancy, nursing, diagnosis of pathological conditions, chronic drug treatment, medical history of abdominal surgery, and OMT in the last week. A comprehensive clinical history was obtained to identify any active or relevant clinical conditions.

The study was performed at the Campus Bio Medico University and Teaching Hospital in Rome, Italy. A total of 71 healthy volunteers were screened from September to November 2015. Four participants were considered ineligible. The remaining participants $(n=67)$ were randomized into the 3 trial arms (ie, experimental, sham therapy, and control groups), but a participant belonging to the experimental group abandoned before the intervention because he or she was intolerant to the osteopathic tests.

The choice of recruiting healthy volunteers was guided by the current lack of clear and objective evidence on the impact of osteopathic techniques on the thoracoabdominal diaphragm. Therefore, it seemed logical to assess the effects of OMT on the diaphragm, if any, first in healthy participants.

Figure 1 resumes the study design. The study protocol is recorded in clinicaltrials.gov (NCT02801058).

The double-blinded design of the trial guarantees that both the volunteer and the ultrasonographer were unaware of the randomization group. Furthermore, the operator had no access to the ultrasound measurement. All participants provided written informed consent. The study protocol was approved by the Università Campus Bio-Medico di Roma ethical committee (Prot. Number: 30/17).

\section{Ultrasound Assessment of Diaphragm}

All participants, regardless of the assigned group, received a comprehensive ultrasound evaluation (Exagyne, Echo Control Medical, Angoulême, France) of 


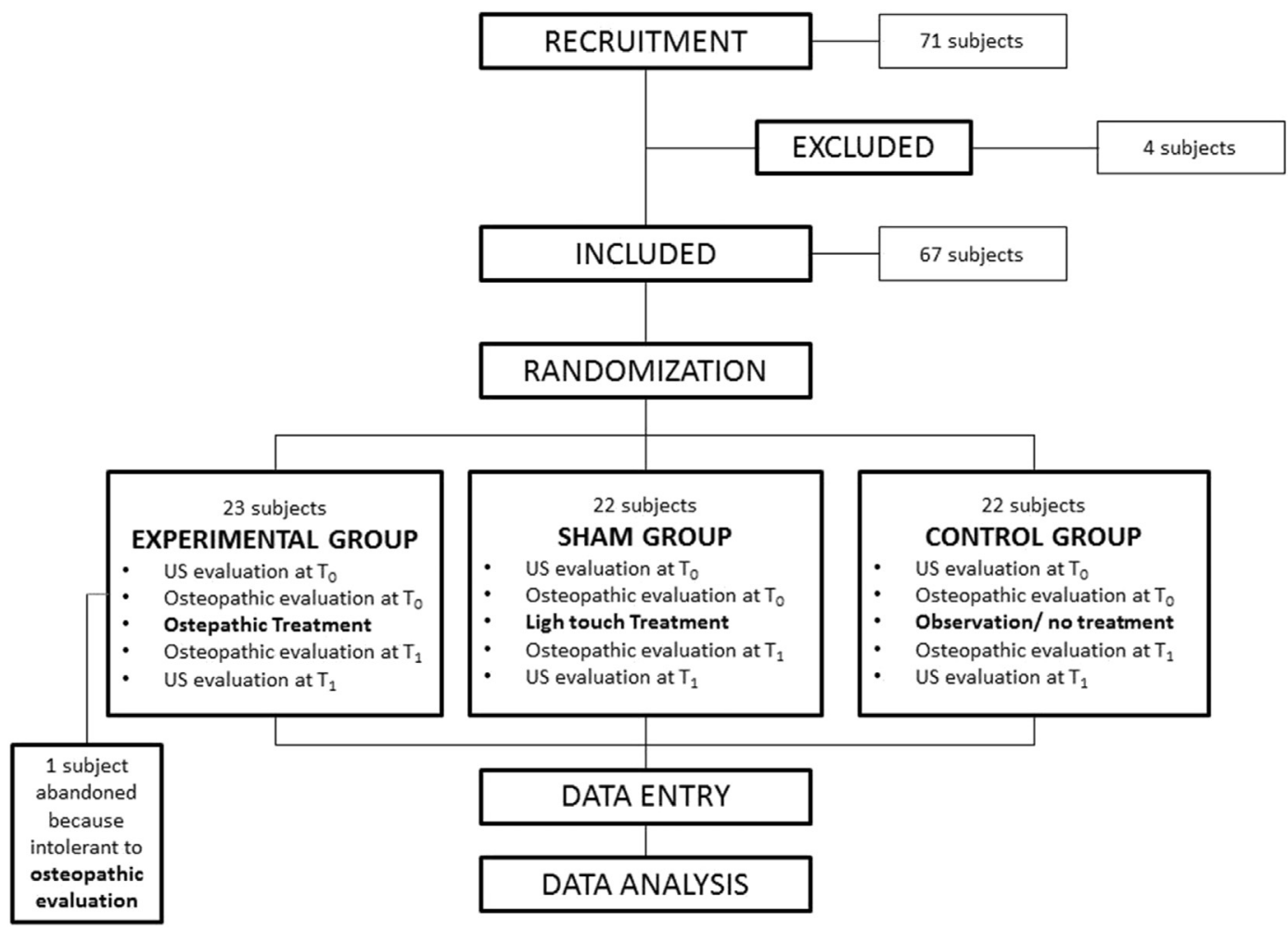

Fig I. Design of the study.

diaphragmatic motion and thickness at the baseline and after intervention. Three consecutive M-mode and Bi-mode measurements of right diaphragm motion were systematically obtained. A subcostal scan using a convex probe and frequencies between 2.5 and $3.5 \mathrm{MHz}$ was achieved during the participant's deep inspiration (inspiratory reserve capacity), starting from functional residual capacity, according to described protocols. ${ }^{18}$ After that, 3 estimations of the diaphragmatic strength by measuring diaphragm thickness at end-inspiration and end-expiration were obtained with a longitudinal scan using a linear probe at $12-\mathrm{MHz}$ frequencies. The thickness of the diaphragm at rest was finally used as a proxy for respiratory muscle trophism. ${ }^{19,20}$ The highest of the 3 obtained values was finally collected.

\section{Osteopathic Evaluation}

An osteopathic evaluation, ${ }^{21}$ testing the domes and pillars of the diaphragm, was performed at the baseline and after intervention. The evaluation protocol is described in the supplementary appendix.

After the intervention, to objectify variations in the degree of somatic dysfunction, a semiquantitative and graduated scale was used according to the following scores: severely worsened somatic dysfunction (1 point), mildly worsened somatic dysfunction (2 points), unchanged somatic dysfunction (3 points), slightly improved somatic dysfunction (4 points), dramatically improved dysfunction (5 points), and complete normalization of somatic dysfunction (6 points).

\section{Interventions}

The control group was simply observed while lying down over the operator's table without receiving any treatment. The sham therapy group received a light touch approach (a gently pressure over the chest and subcostal area, alternate with deep inspirations, without any therapeutic purpose); the experimental group was effectively treated.

The osteopathic techniques ${ }^{22}$ were applied to the diaphragmatic pillars and domes and are described in detail in the supplementary appendix.

\section{Statistical Analysis}

All data are expressed as means or medians ( \pm standard deviation [SD] or $95 \%$ confidence interval [CI]) for continuous variables or as percentages for categorical variables. Sample size calculation was made assuming as clinically significant a change in diaphragmatic motion of at least $12 \mathrm{~mm}$ after treatment, according to a mean described excursion of 37 and $47 \mathrm{~mm}$ in women and men, respectively. ${ }^{16}$ A block size randomization with a 1:1:1 ratio was performed. The osteopath operator (D.M.) was in 
Table I. Demographic and Anthropometric Characteristics of the Study Population

\begin{tabular}{|c|c|c|c|c|c|}
\hline & \multicolumn{3}{|l|}{ Groups } & \multirow[b]{2}{*}{ Total } & \multirow{2}{*}{$\begin{array}{l}P \\
\text { Value }\end{array}$} \\
\hline & Experimental & Sham & Control & & \\
\hline Age, mean (SD) & $41.9(14.6)$ & $377(13.6)$ & $41.7(15.6)$ & $40.4(14.5)$ & .5 \\
\hline BMI mean (SD) & $24.1(2.5)$ & $24.6(4.4)$ & $24.9(4.6)$ & $24.6(3.9)$ & .7 \\
\hline Male sex, n (\%) & $12(54.5)$ & $11(50)$ & $9(40.9)$ & $32(48.5)$ & .6 \\
\hline Smoking, n (\%) & $4(18.2)$ & $7(31.8)$ & $5(22.7)$ & $16(24.2)$ & .5 \\
\hline Sedentary work, n (\%) & $13(59.1)$ & $13(59.1)$ & $12(54.5)$ & $38(57.6)$ & .9 \\
\hline Physically active, n (\%) & $11(50)$ & $12(54.5)$ & $10(45.5)$ & $33(50)$ & .8 \\
\hline
\end{tabular}

$B M I$, body mass index; $S D$, standard deviation.

Table 2. Diaphragmatic Mobility and Thickness Within the Groups at Baseline $\left(T_{0}\right)$ and $T_{1}$

\begin{tabular}{|c|c|c|c|c|c|}
\hline Groups & $\begin{array}{l}\text { Diaphragmatic Mobility } \\
\text { at } \mathrm{T}_{0} \mathrm{~mm}(\mathrm{SD})\end{array}$ & $\begin{array}{l}\text { Diaphragmatic Mobility } \\
\text { at } \mathrm{T}_{1} \mathrm{~mm}(\mathrm{SD})\end{array}$ & $\Delta(\mathrm{mm})$ & $P$ Value & ANOVA \\
\hline Experimental & $68.0(17.9)$ & $82.5(15.8)$ & 14.5 & $<.001$ & $<0.001^{\mathrm{a}}$ \\
\hline Sham & $65.8(10.5)$ & $65.6(11.2)$ & -0.22 & .8 & $>0.05^{\mathrm{b}}$ \\
\hline \multirow[t]{2}{*}{ Control } & 63.7 (17.6) & $61.6(17.1)$ & -2.09 & .7 & $>0.05^{c}$ \\
\hline & $\begin{array}{l}\text { Diaphragmatic Thickness } \\
\text { at } \mathrm{T}_{0}(\mathrm{~mm} ; \mathrm{SD})\end{array}$ & $\begin{array}{l}\text { Diaphragmatic Thickness } \\
\text { at } \mathrm{T}_{1}(\mathrm{~mm} ; \mathrm{SD})\end{array}$ & $\Delta(\mathrm{mm})$ & $P$ Value & ANOVA \\
\hline Experimental & $1.09(0.39)$ & $1.22(0.61)$ & 0.13 & .16 & $>0.05^{\mathrm{a}}$ \\
\hline Sham & $1.13(0.38)$ & $1.19(0.48)$ & 0.06 & .66 & $>0.05^{\mathrm{b}}$ \\
\hline Control & $0.93(0.29)$ & $1.09(0.27)$ & 0.16 & .18 & $>0.05^{\mathrm{c}}$ \\
\hline
\end{tabular}

$A N O V A$, analysis of variance; $S D$, standard deviation.

a vs sham and control.

${ }^{b}$ vs control.

c vs sham.

charge of generating the random allocation sequence, enrolling participants, and assigning participants to interventions. A research randomizer software was used for randomization (iRandomizer version 2.5, Shmoopi LLC, Washington, DC). Comparisons between groups were made by $\chi$-square for categorical variables and Student $t$ test or 1-way analysis of variance (ANOVA) tests followed by Bonferroni post hoc multiple comparison adjustment for continuous variables. The linear relationship was assessed by the Pearson correlation coefficient. All the statistical analyses were performed using SPSS software, version 19.0 (IBM Corp, Armonk, New York).

\section{RESULTS}

Demographics and anthropometric features of the study population are described in Table 1. Among the 66 participants, 32 (48.5\%) were male. The studied population had a mean age of 40.4 (SD 14.5) years and presented a mean body mass index (BMI) of 24.6 (SD 3.9) $\mathrm{kg} / \mathrm{m}^{2}$. Sixteen participants $(24.2 \%)$ were current smokers, 38 $(57.6 \%)$ conducted a sedentary working activity, and only half of them (33 of 66) declared performing regular physical activity. None of these characteristics showed to significantly differ between the 3 randomization groups (Table 1). The baseline diaphragmatic excursion was 68.0 (SD 17.9) $\mathrm{mm}$, without significant differences across groups. A significant increase in diaphragmatic mobility was observed in the experimental group after the osteopathic manipulative techniques (from $68 \mathrm{~mm}$ to $82.5 \mathrm{~mm} ; \Delta=14.5 \mathrm{~mm} ; P<.001$; ANOVA $P<.001$ vs both sham and control groups; Table 2 ). No significant changes were instead observed in the other groups $(\Delta=-0.22 \mathrm{~mm}$ and $-2.09 \mathrm{~mm}$ for the sham and control groups, respectively, $P>.05$; sham vs control ANOVA $P>$ $.05)$. Such increase of mobility was significantly different compared with the modifications that occurred in participants included in the control and the sham groups. 
Table 3. Modifications of Diaphragmatic Mobility in the Experimental Group $(n=22)$ According to Age, BMI, Sex, Smoking Status, Work Activity, and Physical Activity

\begin{tabular}{|c|c|c|c|c|c|}
\hline & $\mathrm{n}$ & Mean at $\mathrm{T}_{0}(\mathrm{SD})$ & Mean at $\mathrm{T}_{1}(\mathrm{SD})$ & $\Delta(\mathrm{mm})$ & $P$ Value \\
\hline Age $<$ median value ${ }^{\mathrm{a}}$ & 11 & $71.6(17.7)$ & $84.3(17.3)$ & 12.7 & .02 \\
\hline Age $>$ median value & 11 & $64.4(18.2)$ & $80.8(14.7)$ & 16.3 & .01 \\
\hline $\mathrm{BMI}<$ median value ${ }^{\mathrm{b}}$ & 11 & $64.5(19.1)$ & $75.7(13.8)$ & 11.1 & .03 \\
\hline $\mathrm{BMI}>$ median value & 11 & $71.5(16.9)$ & $89.4(15.2)$ & 17.9 & .03 \\
\hline Male & 10 & $72.9(15.7)$ & $89.2(12.8)$ & 16.3 & .02 \\
\hline Female & 12 & $62.1(19.4)$ & $74.5(15.8)$ & 12.4 & .03 \\
\hline Current smokers & 4 & $71.1(16.9)$ & $78.0(8.2)$ & 6.9 & .36 \\
\hline Nonsmokers & 18 & $67.3(18.5)$ & $83.5(17.0)$ & 16.2 & .04 \\
\hline Sedentary work & 13 & $63.6(19.3)$ & $78.1(17.5)$ & 14.5 & .08 \\
\hline Nonsedentary work & 9 & $74.4(14.3)$ & $89.0(10.6)$ & 14.6 & .04 \\
\hline Physically active & 11 & $66.8(16.6)$ & $79.9(13.0)$ & 13.1 & .03 \\
\hline Not physically active & 11 & $69.2(19.9)$ & $85.2(18.4)$ & 16.0 & .01 \\
\hline
\end{tabular}

$B M I$, body mass index; $S D$, standard deviation.

${ }^{\mathrm{a}}$ Median age value $=45.5 \mathrm{y}$.

${ }^{\mathrm{b}}$ Median BMI value $=24.0 \mathrm{~kg} / \mathrm{m}^{2}$.

No significant modification was observed for the diaphragmatic thickness after the intervention (Table 2).

Table 3 shows the changes of diaphragmatic mobility according to potential confounders (eg, age, BMI, sex, smoking, sedentary job, and physical activity). Being a current smoker and having a sedentary profession seemed to be associated with a worse response to the osteopathic manipulation of the diaphragm.

As depicted in Figure 2, a strong linear relationship was observed between the diaphragmatic mobility gradient, measured with ultrasonography, and the score assigned by the operator evaluating the change of diaphragm mobility after the manual treatment.

\section{DISCUSSION}

This is the first study to measure the effect of a specific osteopathic manual therapy protocol on the mobility of the thoracic diaphragm. Furthermore, there have only been a limited number of studies investigating the effect of diaphragmatic manipulation in the presence of respiratory diseases such as COPD. ${ }^{23,24}$ In a recent review paper, Heneghan and colleagues ${ }^{24}$ concluded there is currently no evidence to support or reject the use of manipulative therapy for improving lung function in patients with COPD. This view is contradicted by Wearing et al with respect to spinal manipulative therapy and COPD. ${ }^{25}$ Notwithstand- ing, the evidence pertaining to a given clinical condition may not be generalizable to other respiratory conditions or to the healthy participant. ${ }^{26}$

The matching of the groups regarding age, weight, sex, physical activity, job, and smoking exposure minimized the confounding role of these variables in affecting results. Furthermore, our population showed a mean diaphragm excursion of $68 \mathrm{~mm}$ at the baseline, in line with the mean values expected in a general adult population. ${ }^{27,28}$ The absence of any significant change in the diaphragmatic mobility in the sham group can reasonably exclude that a placebo effect might be influencing our findings. On the other hand, it strengthens the significance of the statistically relevant improvement of $14.4 \mathrm{~mm}(+21.3 \%$ compared to the baseline assessment) between the baseline and after OMT in the experimental group.

At variance with age, BMI, sex, and the intensity of physical activity, being a nonsmoker and having a nonsedentary profession might be associated with a better response to the manipulative treatment. The reasons for these finding are unclear, but might lay in the unfavorable effect of smoking and physical inactivity on respiratory muscle training. ${ }^{29}$ In a recent study, adult smokers without airflow obstruction presented with reduced levels of daily physical activity as determined by functional exercise capacity, extended fatigue sensation, anxiety, and depression. ${ }^{30}$ These subgroups of participants might benefit less from a single session of OMT and may instead require multiple and more complex OMT treatments. 


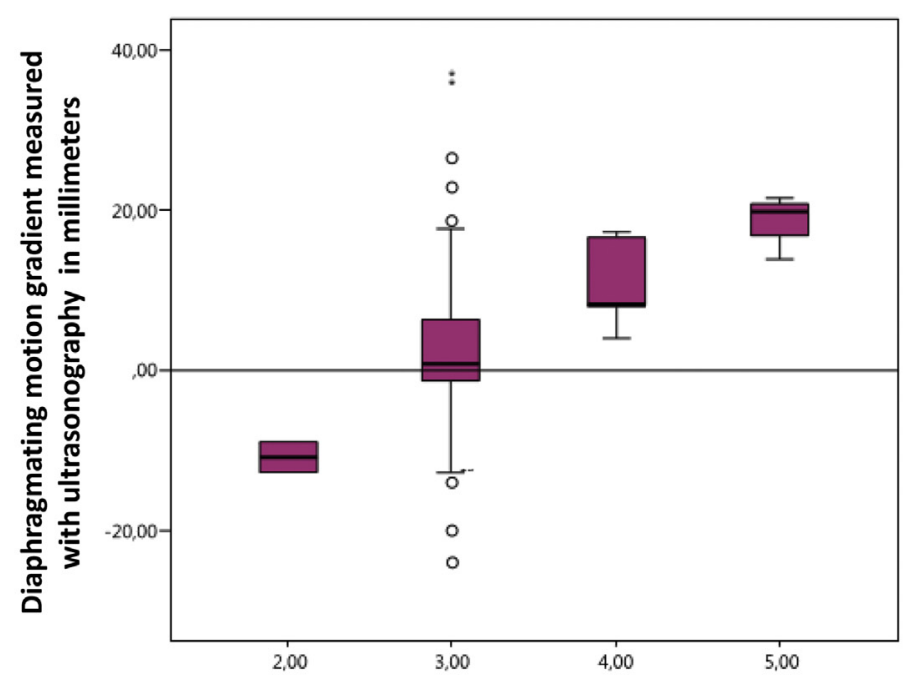

Score assigned by the operator evaluating the change of right hemidiaphragm mobility after intervention

\section{SCORE LEGEND:}

$\mathbf{1}=$ severely worsened somatic dysfunction; $\mathbf{2}=$ mildly worsened somatic dysfunction; 3= unchanged somatic dysfunction; 4= slightly improved somatic dysfunction; $\mathbf{5}=$ dramatically improved dysfunction; $\mathbf{6 =}$ complete normalization of somatic dysfunction.

Fig 2. Box plot showing the relationship between the diaphragmatic mobility gradient measured with ultrasonography and the score assigned by the operator evaluating the change of diaphragm mobility after intervention.

The results reported here support the call for further studies involving groups of healthy people such as athletes where the effect of OMT on performance could be assessed or on professionals who need to control their breathing for specific purposes, such as singers, actors, or teachers. Furthermore, morphologic changes associated with senescence have been shown to reduce the efficiency of the respiratory system. ${ }^{31}$ It would therefore be pertinent to investigate the use of OMT on older people in an attempt to evaluate the potential to improve quality of life, physical performance, and exertional tolerance.

In our study, OMT did not induce any change in diaphragmatic thickness. Although this parameter is correlated with muscle contractility, ${ }^{19}$ it is unlikely that a single application of OMT could be expected to produce a change in diaphragm thickness. Studies specifically designed to investigate the long-term effects of OMT are required to clarify this issue.

Interestingly, there was a relevant correlation between the amplitude of the change in diaphragmatic mobility and the score assigned by the operator evaluating the change after OMT. This finding supports the value of assessing the diaphragm through physical examination. ${ }^{21}$ Although further studies confirming these data and testing the interoperator reliability are called for, this is the first report to correlate the manual feedback with an objective measure such as ultrasound.

\section{Limitations}

The absence of a splenic window for ultrasound evaluation of the left hemidiaphragm did not allow for consideration of specific changes in this section of the diaphragm. Secondly, we were not able to provide any measure of change in lung volumes and flows after OMT because lung function assessments were not routinely obtained. We recommend this type of measure be included in future studies.

In conclusion, the osteopathic techniques used in this study provided beneficial effects on diaphragmatic mobility, but not on thickness, in healthy participants. We hypothesize that further benefits might be derived from repeated and more comprehensive OMT sessions. We recommend that future research involve selected respiratory conditions and include changes in diaphragm mobility and thickness after OMT.

\section{Conclusion}

Osteopathic techniques used in this study improved the diaphragmatic motion in healthy participants. However, muscle thickness did not show statistical significance. 


\section{Funding SOURCES AND CONFlicts of INTEREST}

No funding sources or conflicts of interest were reported for this study.

\section{CONTRIBUTORSHIP INFORMATION}

Concept development (provided idea for the research): D.M., S.S.

Design (planned the methods to generate the results): D.M., S.S.

Supervision (provided oversight, responsible for organization and implementation, writing of the manuscript): D.M., C.L., S.S.

Data collection/processing (responsible for experiments, patient management, organization, or reporting data): D.M., A.M.B., S.S.

Analysis/interpretation (responsible for statistical analysis, evaluation, and presentation of the results): D.M., M.C., R.A.I., S.S.

Literature search (performed the literature search): D.M., S.S.

Writing (responsible for writing a substantive part of the manuscript): D.M., M.C., R.A.I., S.S.

Critical review (revised manuscript for intellectual content, this does not relate to spelling and grammar checking): D.M., M.C., C.L., A.M.B., R.A.I.

\section{APPENDIX A. SUPPLEMENTARY DATA}

Supplementary data to this article can be found online at https://doi.org/10.1016/j.jmpt.2018.08.001.

\section{Practical Applications}

- This is the first randomized controlled trial that evaluated osteopathic manipulation of the diaphragm using diagnostic ultrasound.

- The study showed a significant increase in diaphragmatic mobility after the application of a single osteopathic manipulation on healthy participants.

\section{REFERENCES}

1. Bordoni B, Zanier E. Anatomic connections of the diaphragm: influence of respiration on the body system. $J$ Multidiscip Healthc. 2013;6:281-291.
2. Taylor EW, Jordan D, Coote JH. Central control of the cardiovascular and respiratory systems and their interactions in vertebrates. Physiol Rev. 1999;79(3):855-916.

3. Gignon L, Roger C, Bastide S, et al. Influence of diaphragmatic motion on inferior vena cava diameter respiratory variations in healthy volunteers. Anesthesiology. 2016;124(6):1338-1346.

4. Kimura BJ, Dalugdugan R, Gilcrease III GW, Phan JN, Showalter BK, Wolfson T. The effect of breathing manner on inferior vena caval diameter. Eur J Echocardiogr. 2011;12 (2):120-123.

5. Yasuma F, Hayano J. Respiratory sinus arrhythmia: why does the heartbeat synchronize with respiratory rhythm? Chest. 2004;125(2):683-690.

6. Degenhardt BF, Kuchera ML. Update on osteopathic medical concepts and the lymphatic system. J Am Osteopath Assoc. 1996;96(2):97-100.

7. Wallace E, McPartland JM, Jones JM, Kuchera WA, Buser BR. Lymphatic system: lymphatic manipulative techniques. In: Ward RC, ed. Foundations for Osteopathic Medicine. 2nd ed. London, UK: Lippincott Williams \& Wilkins; 2003.

8. World Osteopathic Health Organization. Osteopathic glossary. Available at: www.woho.org. Accessed April 19, 2008.

9. World Health Organization (WHO). Benchmarks for training in osteopathy, WHO, Geneva. Available at: https://www.who.int/ medicines/areas/traditional/BenchmarksforTraininginOsteopathy. pdf. Accessed February 28, 2019.

10. da Silva RC, de Sá CC, Pascual-Vaca ÁO, et al. Increase of lower esophageal sphincter pressure after osteopathic intervention on the diaphragm in patients with gastroesophageal reflux. Dis Esophagus. 2013;26(5):451-456.

11. Noll DR, Degenhardt BF, Morley TF, et al. Efficacy of osteopathic manipulation as an adjunctive treatment for hospitalized patients with pneumonia: a randomized controlled trial. Osteopath Med Prim Care. 2010;4:2.

12. Noll DR, Degenhardt BF, Johnson JC, Burt SA. Immediate effects of osteopathic manipulative treatment in elderly patients with chronic obstructive pulmonary disease. $J \mathrm{Am}$ Osteopath Assoc. 2008;108(5):251-259.

13. Zanotti E, Berardinelli P, Bizzarri C, et al. Osteopathic manipulative treatment effectiveness in severe chronic obstructive pulmonary disease: a pilot study. Complement Ther Med. 2012;20(1-2):16-22.

14. Cruz-Montecinos C, Godoy-Olave D, Contreras-Briceño FA, et al. The immediate effect of soft tissue manual therapy intervention on lung function in severe chronic obstructive pulmonary disease. Int J Chron Obstruct Pulmon Dis. 2017;12:691-696.

15. Engel RM, Gonski P, Beath K, Vemulpad S. Medium term effects of including manual therapy in a pulmonary rehabilitation program for chronic obstructive pulmonary disease (COPD): a randomized controlled pilot trial. J Man Manip Ther. 2016;24(2):80-89.

16. Summerhill EM, El-Sameed YA, Glidden TJ, McCool FD. Monitoring recovery from diaphragm paralysis with ultrasound. Chest. 2008;133(3):737-743.

17. Sferrazza Papa GF, Pellegrino GM, Di Marco F, et al. A review of the ultrasound assessment of diaphragmatic function in clinical practice. Respiration. 2016;91(5):403-411.

18. Boussuges A, Gole Y, Blanc P. Diaphragmatic motion studied by m-mode ultrasonography: methods, reproducibility, and normal values. Chest. 2009;135(2):391-400.

19. Cohn D, Benditt JO, Eveloff S, McCool FD. Diaphragm thickening during inspiration. J Appl Physiol (1985). 1997;83 (1):291-296.

20. Baria MR, Shahgholi L, Sorenson EJ, et al. B-mode ultrasound assessment of diaphragm structure and function in patients with COPD. Chest. 2014;146(3):680-685. 
21. Bordoni B, Marelli F, Morabito B, Sacconi B. Manual evaluation of the diaphragm muscle. Int J Chron Obstruct Pulmon Dis. 2016;11:1949-1956.

22. Pagliaro R. Osteopatia in campo viscerale-L'addome. Marrapese Editore Roma; 2006.

23. Rocha T, Souza H, Brandão DC, et al. The Manual Diaphragm Release Technique improves diaphragmatic mobility, inspiratory capacity and exercise capacity in people with chronic obstructive pulmonary disease: a randomised trial. $J$ Physiother. 2015;61(4):182-189.

24. Heneghan NR, Adab P, Balanos GM, Jordan RE. Manual therapy for chronic obstructive airways disease: a systematic review of current evidence. Man Ther. 2012;17(6):507-518.

25. Wearing J, Beaumont S, Forbes D, Brown B, Engel R. The use of spinal manipulative therapy in the management of chronic obstructive pulmonary disease: a systematic review. $J$ Altern Complement Med. 2016;22(2):108-114.

26. De Troyer A. Effectof hyperinflation on the diaphragm. Eur Respir J. 1997;10(3):708-713.
27. Cohen E, Mier A, Heywood P, Murphy K, Boultbee J, Guz A. Excursion-volume relation of the right hemidiaphragm measured by ultrasonography and respiratory airflow measurements. Thorax. 1994;49(9):885-889.

28. Targhetta R, Chavagneux R, Ayoub J, et al. Cinétique diaphragmatiquedroitemesurée par ultrasonographieen mode TM avec spirométrieconcomitante chez le sujet normal et asthmatique, Résultatspréliminaries. Rev Med Interne. 1995; 16(11):819-826.

29. Jayes L, Haslam PL, Gratziou CG, et al. SmokeHaz: systematic reviews and meta-analyses of the effects of smoking on respiratory health. Chest. 2016;150:164-179.

30. Le Neindre A, Mongodi S, Philippart F, Bouhemad B. Thoracic ultrasound: potential new tool for physiotherapists in respiratory management. A narrative review. J Crit Care. 2016;31(1):101-109.

31. Lalley PM. The aging respiratory system — pulmonary structure, function and neural control. Respir Physiol Neurobiol. 2013; 187(3):199-210. 